Working Paper 03-50

Economics Series 19

October 2003
Departamento de Economía

Universidad Carlos III de Madrid

Calle Madrid, 126

28903 Getafe (Spain)

Fax (34) 916249875

\title{
EFFICIENCY IN A MATCHING MODEL WITH HETEROGENEOUS AGENTS: TOO MANY GOOD OR BAD JOBS? *
}

\author{
Maite Blázquez ${ }^{1}$ and Marcel Jansen ${ }^{2}$
}

\begin{abstract}
This paper analyzes the efficiency of the equilibrium allocation in a matching model with two types of workers and jobs. The technology is such that high-skill workers can perform all jobs, while low-skill workers can only perform unskilled jobs. In this setup two types equilibria may occur. A "cross-skill matching equilibrium" in which high-skill workers accept all jobs and an "ex-post segmentation equilibrium" in which they accept only skilled jobs. Our first result shows that the equilibrium with ex-post bargaining is never efficient. Second, under Hosios" (1990) condition we show that low-skill workers are overvalued, while the opposite holds for high-skill workers. In equilibrium, firms therefore create too few unskilled jobs and too many skilled jobs. In addition, high-skill workers may decide to accept unskilled jobs while the efficient allocation features ex-post segmentation. Finally, in an extension we show that efficiency can be restored through taxation and we analyze how workers' bargaining strength affects unemployment and the degree of skill-mismatch.
\end{abstract}

Keywords: matching, ex post bargaining, heterogeneity, efficiency.

JEL Classification: C78, D61, J64.

${ }^{1}$ M. Blázquez, Departamento de Economía, Universidad Carlos III de Madrid; E.mail: maite blazquez@yahoo.es. Phone: (34) 916249781

2 M. Jansen, Departamento de Economía, Universidad Carlos III de Madrid. E.mail: jansen@eco.uc3m.es. Phone: (34) 916245740

\footnotetext{
* We are grateful to Juan Dolado, Barbara Petrongolo, Victor Rios-Rull, Tim Kehou and Michele Boldrin and seminar participants at Universidad Carlos III de Madrid and the 2003 Workshop on Dynamic Macroeconomics in Vigo for useful comments and suggestions. The usual disclaimer applies.
} 


\section{Introduction}

This paper analyzes the efficiency of the resource allocation in a matching model with two types of workers and jobs. Workers' skills are exogenous and we assume that high-skill workers can do both jobs, skilled and unskilled, while low-skill workers can only perform unskilled jobs. This setup, first introduced by Albrecht and Vroman (2002), is useful to study phenomena like skill-biased technological change or low-wage competition. However, so far little is known about the efficiency properties of these models.

Our objective in this paper is to characterise the entire set of possible steady state equilibria with random search and ex post bargaining. As in Albrecht and Vroman we therefore need to distinguish between two types of equilibria. A cross-skill matching equilibrium in which high-skill workers accept both types of jobs and an ex post segmentation equilibrium in which these workers only match with skilled jobs. To achieve this goal we compare the equilibrium with the efficient allocation chosen by a social planner. This planner controls the mass of the two types of vacancies and the optimal matching rule for high-skill workers and her objective is to maximise the value of net-output.

Our first result shows that the equilibrium with ex post bargaining is never efficient. Second, under Hosios' (1990) condition we show that highskill workers are undervalued in equilibrium while the opposite holds for low-skill workers. In a cross-skill matching equilibrium this results in an insufficient number of unskilled jobs as firms need to pay low-skill workers more than their shadow value. Furthermore, due to the undervaluation of high-skill workers, there may be cases in which these workers accept unskilled jobs while the efficient allocation features ex post segmentation.

The result that the labour market tends to create too few unskilled jobs achieves our first goal. Namely, to show that the presence of high-skill workers 
tends to harm low-skill workers by raising their unemployment rate above the socially optimal level. Furthermore, our results suggest that this distortion becomes even stronger when we move from cross-skill matching to ex-post segmentation. In the latter case high-skill workers no longer occupy unskilled jobs, but this effect is more than offset by a discrete fall in the supply of these jobs. Finally, it should be noted that our model can also generate the opposite result, i.e. over-creation of unskilled jobs. The latter occurs when workers' surplus share is sufficiently smaller than the Hosios' value. In this parameter region, firms create too many jobs and a suboptimally large share of these jobs is unskilled. In contrast, for sufficiently high values of workers' bargaining power, the fraction of unskilled jobs is above the efficient value, but because the overall number of vacancies per job seeker is too low, the unemployment rate of low-skill workers is still suboptimally high.

To understand the intuition behind our results, it is convenient to make a comparison with the results of Hosios (1990). For an environment with ex ante homogeneous agents he demonstrated that the equilibrium is efficient if workers' bargaining power is equal to the elasticity of the matching function with respect to the mass of unemployed workers. In that case, the search externalities are perfectly internalised in the wage and unemployed workers' expected income coincides with their shadow value.

In contrast, in our economy an additional unemployed worker congests the market for workers with different skill levels. The absolute value of this externality is the same for both types of workers. However, in the case of a high-skill worker it corresponds to a lower share of her expected future productivity than in the case of a low-skill worker. When Hosios' condition is satisfied, high-skill workers are therefore under-valued in equilibrium while the opposite holds for low-skill workers.

The above argument seems to suggest that there may exist a pair of surplus shares, one for each type of worker, that decentralises the efficient 
allocation. However, this is not the case. In our model Hosios' condition is necessary to ensure that firms create the right number of jobs per job seeker. When we introduce different bargaining strengths to correct the distortion of the job distribution - the over-creation of skilled jobs and the undercreation of unskilled jobs - we would therefore end up with an inefficient number of jobs. ${ }^{1}$ Instead, it is easy to show that the efficient allocation can be decentralised through taxation. In the paper we analyse the case of lump sum taxes on unemployed workers, showing that the government should levy a tax on unemployed workers with a low skill level, while workers with a high skill level should receive a subsidy during unemployment. The proposed tax scheme confirms our explanation about the source of the over-valuation (under-valuation) of low-skill (high-skill) workers, but it may be difficult to implement. An alternative would be to levy a hiring tax on skilled jobs. Also in this case, the relative profits of skilled jobs go down, but the hiring tax on skilled jobs avoids the negative income effect on low-skill workers.

\subsection{Related literature}

The seminal contribution on efficiency with heterogeneous agents is Sattinger (1995). In his model there is a fixed supply of $T$ types of workers and firms whose matching rates are fixed. Sattinger's main result shows that heterogeneity may give rise to multiple and inefficient equilibria. Like us, he also shows that the equilibrium payoffs never coincide with the shadow value of agents. However, while computing the shadow value of a worker, Sattinger sterilises the effects on the other workers by increasing the job supply to offset any changes in matching probabilities. Distortion of the wage payments therefore arise because workers ignore the costs of the firms with whom they interview and not because of the congestion effects on other workers as in our

\footnotetext{
${ }^{1}$ The details of the proof are available upon request.
} 
case. Our work is therefore more closely related to Shimer and Smith (2001a, 2001b) who use a social planner problem to derive the efficient allocations in an environment with assortative matching and endogeneous search intensity. In Shimer and Smith (2001b) the aim is to show that the model may have efficient non-stationary allocations. Our work is closer in spirit to Shimer and Smith (2001a). In this paper the authors show that the decentralised equilibrium is never efficient without search subsidies. In particular, in the decentralised equilibrium without subsidies, the most productive agents do not search hard enough and they accept too many (low-productivity) matches. In contrast, low-productivity types search too hard and they reject too many matches. An optimal tax scheme therefore involves a search subsidy for the high-productivity agents and a search penalty for the low-productivity workers.

This last result is somewhat similar to our optimal tax scheme. However, the advantage of our simple model is that we can immediately relate our results to the well-known results of Hosios. Hence, while Shimer and Smith (2002a) obtain the inefficiency of the resource allocation via the derivation of a non-trivial tax scheme, we are able to prove that low-skill workers are over-valued in equilibrium. Moreover, our results indicate that this is a general feature of models with ex ante heterogeneous agents. One of the main contributions of this paper is therefore that we provide a clear intuition for the inefficiency of the resource allocation in economies with heterogeneous agents and ex post bargaining. Finally, Shimer and Smith (2002a) consider an economy with fixed pool of agents, while we allow for free entry of firms.

The outline of the rest of the paper is as follows. Section 2 introduces the model. The next section derives the two possible equilibrium configurations. The set of efficient allocation is derived in Section 4. This section starts with a characterisation of efficient cross-skill matching allocations. Next, we discuss the efficient allocations with ex post segmentation and at the end of 
the section we derive the optimal matching rule of high-skill workers, showing that high-skill workers may accept unskilled jobs while the efficient allocation features ex post segmentation. Finally, in section 5 we derive the optimal taxes and we show how extreme values of the bargaining strength may lead to overcreation of unskilled jobs.

\section{The Model}

\subsection{Basic Assumptions}

Consider an economy populated by a continuum of risk-neutral workers with measure normalised to unity. The distribution of workers' abilities is exogenous. Specifically, we assume that a fraction $\mu \in(0,1)$ of the workers is low-skill $(l)$, while the remaining fraction $1-\mu$ is high-skill $(h)$. All workers are infinitely-live and time is continuous.

There are two types of jobs: skilled jobs $(s)$, and unskilled jobs $(u)$. The technology is such that, unskilled jobs can be done by either type of workers, while skilled jobs can only be done by high-skill workers. Furthermore, we assume that high-skill workers are more productive when matched with skilled jobs, while both types of workers are equally productive in unskilled jobs. Formally, let $y(i, j)$ define the flow output of a job $j(=u, s)$ that is filled by a worker of type $i(=l, h)$. Our assumptions on the production technology can then be summarised as follows:

$$
y(h, s)=y(s)>y(h, u)=y(l, u)=y(u)>y(l, s)=0
$$

For convenience, we assume that firms can open at most one job. The choice of the type of job is irreversible, and the mass of each type of job is determined by a free-entry condition. 


\subsection{Matching}

Unemployed workers and vacancies are assumed to meet each other randomly. The total number of matches between a worker and firm is determined by a constant returns to scale matching function.

$$
X=X(v, u)
$$

where $v$ is the mass of vacancies and $u$ is the mass of unemployed workers. We assume that $X(.,$.$) is strictly increasing in both arguments and we denote$ the skilled labour market tightness by $\theta=\frac{v}{u}$. Let $\phi$ denote the fraction of unskilled vacancies, and let $\eta$ be the fraction of low-skill workers among unemployed. Accordingly, the rate at which skilled vacancies are filled is given by $(1-\eta) q(\theta)=(1-\eta) X\left(1, \frac{1}{\theta}\right)$. Similarly, the matching rate for workers is denoted by $\theta q(\theta)$, but since low-skill workers are not qualified for skilled jobs their effective arrival rate will be $\phi \theta q(\theta)$. The properties of the matching function imply that the matching rate of workers (firms) is increasing (decreasing) in $\theta$ and we assume

$$
\lim _{\theta \rightarrow 0} \theta q(\theta)=\lim _{\theta \rightarrow \infty} q(\theta)=0 \text { and } \lim _{\theta \rightarrow 0} q(\theta)=\lim _{\theta \rightarrow \infty} \theta q(\theta)=\infty .
$$

In particular, we are going to analyze the case of a Cobb-Douglas matching function:

$$
X=u^{\alpha} v^{1-\alpha}
$$

The meeting process between workers and vacancies is assumed to be undirected, in the sense that a low-skill worker encounters a skilled vacancy (and thus is unable to consummate the match) with a probability per unit 
of time that is proportional to the fraction of skilled vacancies. Similarly, a high-skill worker finds an unskilled vacancy with a probability per unit of time that is proportional to the fraction of unskilled vacancies. In this manner, we capture the idea that, given the overall labour market conditions, low-skill workers are better off the greater the fraction of unskilled vacancies, and vice versa for high-skill workers. Similarly, all else equal, a firm with an unskilled vacancy is better off the greater the fraction of job seekers that are low-skill.

\subsection{Wages and Asset Values}

When a match is formed, the firm-worker pair divides the surplus of the match according to the Nash bargaining solution. The worker's share of the surplus is denoted by $\beta \in(0,1)$. Let $U(i)$ be the value of an unemployed worker of type $i$, and let $V(j)$ denote the value of a vacant job of type $j$. We also define $W(i, j)$ as the value of employment for a worker of type $i$ on a job of type $j$, and $J(i, j)$ as the value of a type $j$ job filled by a worker of type $i$. Accordingly, the surplus generated by a match between a worker of type $i$ and a job of type $j$ is given by: $S(i, j)=W(i, j)+J(i, j)-V(j)-U(i)$, and the corresponding wage $w(i, j)$ solves the Nash bargaining solution ${ }^{2}$ :

$$
(1-\beta)[W(i, j)-U(i)]=\beta[J(i, j)-V(j)]
$$

We now continue with the derivation of the asset value equations for workers. Let $r$ be the common discount rate for both firms and workers . Job destruction is exogenous and follows a Poisson process with arrival rate $s$ that is common for both jobs. Whenever a job is destroyed, the worker becomes unemployed while the job becomes vacant. Let $b<y(u)$ denote the flow income of an unemployed worker, which is assumed to be equal for both

\footnotetext{
${ }^{2} \mathrm{~A}$ match between a worker of type $i$ and a job of type $j$ will be formed if and only if $S(i, j) \geq 0$
} 
types of workers, and which is to be interpreted as home production without need of any tax-financing scheme.

$$
r U(h)=b+\theta^{1-\alpha}\{\phi \max [W(h, u)-U(h), 0]+(1-\phi)[W(h, s)-U(h)]\}
$$

$$
\begin{gathered}
r W(h, s)=w(h, s)-s[W(h, s)-U(h)] \\
r W(h, u)=w(h, u)-s[W(h, u)-U(h)] \\
r U(l)=b+\theta^{1-\alpha} \phi[W(l, u)-U(l)] \\
r W(l, u)=w(l, u)-s[W(l, u)-U(l)]
\end{gathered}
$$

Equation (2) shows that high-skill workers are capable of undertaking either skilled or unskilled jobs. This is why the value associated with the arrival of an unskilled job is $\max [W(h, u)-U(h), 0]$. In contrast, equation (5) reflects the fact that low-skill workers can only match with unskilled jobs.

Similarly, we derive the asset value equations for filled and unfilled jobs. Let $\gamma$ denote the cost, per unit of time of maintaining an unfilled job. The values of skilled and unskilled vacancies, denoted by $V(s)$ and $V(u)$ respectively, are then given by:

$$
r V(s)=-\gamma+\theta^{-\alpha}(1-\eta)[J(h, s)-V(s)]
$$




$$
r V(u)=-\gamma+\theta^{-\alpha}\{\eta[J(l, u)-V(u)]+(1-\eta) \max [J(h, u)-V(u), 0]\}
$$

Equation (7) reflects the fact that skilled vacancies can only be filled with high-skill workers. However, the value of an unskilled vacancy, given by equation (8), reflects the assumption that while both worker types can perform unskilled jobs, it may not be worthwhile for high-skill workers to accept unskilled jobs. That is why the value of meeting a high-skill worker is $\max [J(h, u)-V(u), 0]$.

The value to a firm of having an unskilled vacancy filled by either low, or high-skill workers, is denoted by $J(l, u)$ and $J(h, u)$, respectively, and satisfies:

$$
\begin{gathered}
r J(h, u)=y(u)-w(h, u)-s[J(h, u)-V(u)] \\
r J(l, u)=y(u)-w(l, u)-s[J(l, u)-V(u)]
\end{gathered}
$$

while the value to the firm of a skilled job filled by a high-skill worker is:

$$
r J(h, s)=y(s)-w(h, s)-s[J(h, s)-V(s)]
$$

Substituting equations (2) - (11) into (1), and taking into account that, in steady state equilibrium, the free entry condition $V(j)=0$ must be satisfied for $j \in(u, s)$, we obtain the expression for wages:

$$
w(i, j)=r U(i)+\beta[y(j)-r U(i)]
$$




\section{Equilibrium}

The analysis of the equilibrium allocation of this type of models is completely developed by $\mathrm{A} \& \mathrm{~V}(2002)$.

\subsection{Cross-skill Matching}

We begin with the derivation of the equilibrium when the matches between high-skill workers and unskilled vacancies are mutually beneficial and, therefore, consummated. We refer to this situation as the "cross-skill matching" equilibrium. The equilibrium is characterized by a vector of endogenous variables $\{\theta, \phi, \eta, u\}$ satisfying: ( $i$ ) two steady state conditions and, (ii) two free entry conditions.

The steady state conditions require that the flows into and out of unemployment for both types of workers must be equal:

$$
\begin{gathered}
\phi \theta^{1-\alpha} \eta u=s[\mu-\eta u] \\
\theta^{1-\alpha}(1-\eta) u=s[1-\mu-(1-\eta) u]
\end{gathered}
$$

where $\phi \theta^{1-\alpha} \eta u$ defines the flow of low-skill workers out of unemployment and $s[\mu-\eta u]$ denotes the corresponding flow into unemployment. Similarly, for high-skill workers, $\theta^{1-\alpha}(1-\eta) u$ denotes the flow of high-skill workers out of unemployment, and $s[1-\mu-(1-\eta) u]$ the corresponding flow into unemployment.

The two steady-state conditions can be solved for $\phi$ and $u$ in terms of $\theta$ and $\eta$. This yields:

$$
\begin{gathered}
\phi=\frac{\mu(1-\eta) \theta^{1-\alpha}+(\mu-\eta) s}{(1-\mu) \eta \theta^{1-\alpha}} \\
u=\frac{s(1-\mu)}{\left[s+\theta^{1-\alpha}\right](1-\eta)}
\end{gathered}
$$


Notice that $\phi$ is decreasing in $\eta$ and increasing in $\theta$, the latter so long as $\eta>\mu$. This condition is necessarily satisfied in a cross skill matching equilibrium since low-skill workers exit unemployment at a lower rate than high-skill workers. Their share in the pool of unemployed workers is therefore larger than their share in the population.

The other two equations that characterize a cross-skill matching equilibrium come from the two free entry conditions. As it turns out, however, it is more convenient to work with the equivalent conditions $V(u)=V(s)=0$. Using equations (7), (11) and (12), the free entry condition for skilled vacancies can be written as:

$$
\gamma=\theta^{-\alpha}(1-\eta)(1-\beta)\left[\frac{[y(s)-r U(h)]}{r+s}\right]
$$

Similarly, substituting (9), (10) and (12) into (8), the free entry condition for unskilled vacancies becomes:

$$
\gamma=\theta^{-\alpha}(1-\beta)\left\{\eta\left[\frac{[y(u)-r U(l)]}{r+s}\right]+(1-\eta)\left[\frac{[y(u)-r U(h)]}{r+s}\right]\right\}
$$

where the outside options for both high-, and low-skill workers, $r U(h)$ and $r U(l)$, are given by the following expressions:

$$
\begin{gathered}
r U(h)=\frac{b(r+s)+\beta \theta^{1-\alpha}[\phi y(u)+(1-\phi) y(s)]}{r+s+\beta \theta^{1-\alpha}} \\
r U(l)=\frac{b(r+s)+\beta \phi \theta^{1-\alpha} y(u)}{r+s+\beta \phi \theta^{1-\alpha}}
\end{gathered}
$$

Making use of the "equal-value condition", $V(s)=V(u)$, we obtain the third equation that characterizes the decentralized equilibrium:

$$
\left(r+s+\beta \phi \theta^{1-\alpha}\right)=\frac{\eta(r+s)(y(u)-b)}{(1-\eta)(y(s)-y(u))}
$$


The fourth and last equation is obtained by substitution of (21) into the free entry condition for skilled vacancies:

$$
\gamma\left(r+s+\beta \theta^{1-\alpha}\right)=(1-\beta) \theta^{-\alpha}(y(u)-b)
$$

Given our assumption about the matching technology, equation (22) defines a unique solution for $\theta$. Also, from equation (22) it can be observed that neither changes in the skill distribution of the population, $\mu$, nor changes in the productivity of skilled jobs, $y(s)$, affect the equilibrium value of the labour market tightness.

Definition 1 A cross-skill matching equilibrium can be summarized by a vector $\left\{\theta_{E}, \phi_{E}, \eta_{E}, u_{E}\right\}$ satisfying equations (15), (16), (21) and (22).

A match will be formed if the surplus of the match is positive, $W(i, j)+$ $J(i, j) \geq V(j)+U(i)$. Thus, a cross-skill matching equilibrium exists whenever it is worthwhile for high-skill workers to take unskilled jobs. From equation (18), this will occur if and only if $S(h, u)=[y(u)-r U(h)] /(r+s) \geq 0$, and so we obtain the following condition for a cross-skill matching equilibrium:

$$
y(u)-r U(h) \geq 0
$$

Equation (23) implies that, in order for a cross-skill matching equilibrium to be feasible, the flow output of an unskilled job filled by a high-skill worker must be greater or equal than the expected lifetime income for this type of worker.

Finally, before turning to the ex post segmentation equilibrium, we need to rule out the corner solution in which firms only supply unskilled jobs. In this situation only unskilled vacancies are offered, $\phi=1$, that is, the value of opening skilled vacancies must be negative. The condition on the parameters 
that ensures this is the following (see Albrecht and Vroman (2002))

$$
y(u)-b>(1-\mu)\left[y(s)-b+\frac{\beta\left(\theta^{*}\right)^{1-\alpha}(y(s)-y(u))}{r+s}\right]
$$

where $\theta^{*}$ is the value of $\theta$ that solves equation (22). Thus, in order to avoid the corner solution we need that skilled vacancies, filled with high-skill workers, are sufficiently productive.

\subsection{Ex Post Segmentation}

In this case, high-skill workers only match with skilled vacancies, that is $y(u)-r U(h)<0$. The two flow conditions now become as follows:

$$
\begin{gathered}
\phi \theta^{1-\alpha} \eta u=s(\mu-\eta u) \\
(1-\phi) \theta^{1-\alpha}(1-\eta) u=s[(1-\mu)-(1-\eta) u]
\end{gathered}
$$

Notice that the steady-state flow condition for low-skill workers is the same as in the cross-skill matching equilibrium. For high-skill workers, on the contrary, the exit rate out of unemployment is now reduced to $(1-\phi) \theta^{1-\alpha}$.

Finally, following the same reasoning as in the cross-skill matching case, the two free entry conditions associated with ex post segmentation satisfy:

$$
\begin{gathered}
\gamma=\theta^{-\alpha} \eta(1-\beta)\left[\frac{[y(u)-b]}{r+s+\beta \phi \theta^{1-\alpha}}\right] \\
\gamma=\theta^{-\alpha}(1-\eta)(1-\beta)\left[\frac{[y(s)-b]}{r+s+\beta(1-\phi) \theta^{1-\alpha}}\right]
\end{gathered}
$$

The free-entry condition for unskilled vacancies, given by equation (27), is decreasing in $\theta$ and increasing $\eta$, the latter because, by equation (25), it can be shown that $\phi$ is decreasing in $\eta$. On the contrary, the free entry 
condition for skilled vacancies, equation (28), is decreasing in both $\theta$ and $\eta$. Thus, increases in the labour market tightness reduce the value of both types of vacancies. On the other hand, all else equal, as the fraction of low-skilled among unemployed increases, the value of unskilled (skilled) vacancies increases (decreases). Therefore, equation $V(u)=0$ defines an upward curve in the positive quadrant $(\theta, \eta)$, while equation $V(s)=0$ defines a downward curve in the same quadrant. The intersection of both loci, therefore, determine a unique ex post segmentation equilibrium (Figure 1).

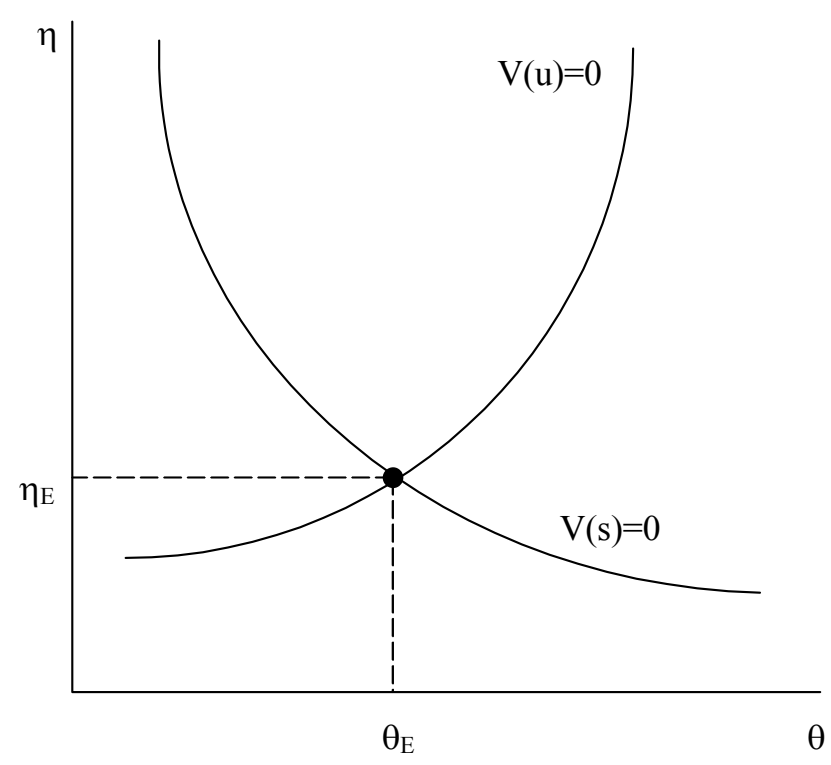

Figure 1: Equilibrium

Thus, if the parameters of the model are consistent with ex post segmentation, there will be a unique equilibrium of this type. The same reasoning applies to the cross-skill matching equilibrium. However, there may be some range of parameter values in which both types of equilibria coexist. As pointed out by Albrecht and Vroman (2002) multiple equilibria can be generated by a coordination externality. Nonetheless, multiple equilibria do not occur for all possible parameter configurations. In particular, for an intermediate range of parameter values, there are two pure-strategy equilibrium 
possibilities, one with cross-skill matching and one with ex post segmentation $^{3}$.

\section{Efficient Allocation}

We now turn to the welfare properties of the model. The efficient allocation is derived using the construct of a "social planner" who chooses the time path of the masses of vacancies, $v(s)$ and $v(u)$, and unemployed workers, $u(h)$ and $u(l)$, to maximize the value of net output. Let $Y(i, j)=y(i, j) /(r+s)$ be the expected productivity of a match between a worker of type $i \in(l, h)$ and a job of type $j \in(u, s)$. Finally, let $R_{t} \in[0,1] \forall t$ define the matching rule for high-skill workers and unskilled vacancies. If $R_{t}$ takes value 1 , then high-skill workers should always accept matches with unskilled vacancies (cross-skill matching), while a value of 0 means that high-skill workers should never accept unskilled job offers (ex-post segmentation). For any $R_{t} \in(0,1)$ we would have a mixed matching strategy.

The social planner's problem can succinctly be written as:

$$
\begin{gathered}
\max _{\theta_{t}, \phi_{t}, u_{t}, R_{t}} \int_{0}^{\infty}\left\{\theta_{t}^{1-\alpha}\left[u(h)_{t}\left[R_{t} \phi_{t} Y(h, u)+\left(1-\phi_{t}\right) Y(h, s)\right]+u(l)_{t} \phi_{t} Y(l, u)\right]+\right. \\
\left.+b\left(u(h)_{t}+u(l)_{t}\right)-\gamma \theta_{t}\left(u(h)_{t}+u(l) t\right)\right\} e^{-r t} d t
\end{gathered}
$$

s.t

$$
\begin{gathered}
\dot{u}(l)_{t}=s\left[\mu-u(l)_{t}\right]-\left[\phi_{t} \theta_{t}^{1-\alpha}\right] u(l)_{t} \\
\dot{u}(h)_{t}=s\left[1-\mu-u(h)_{t}\right]-\left[\theta_{t}^{1-\alpha}\right] u(h)_{t}\left[R_{t}+\left(1-R_{t}\right)\left(1-\phi_{t}\right)\right]
\end{gathered}
$$

\footnotetext{
${ }^{3}$ See Albrecht and Vroman (2002) for details.
} 
In Appendix A we solve for the steady state efficient allocation using the optimal control.theory Henceforth, we suppress all time subscripts. Furthermore, from the optimality condition for $R$, it follows immediately that the optimal matching rule depends on the sign of $y(u)-(r+s) \lambda(h)$, where $\lambda(h)$ is the shadow value of a high-skill worker:

$$
R=\left\{\begin{array}{c}
1 \quad \text { if } \quad y(u)>(r+s) \lambda(h) \\
\in[0,1] \text { if } y(u)=(r+s) \lambda(h) \\
0 \quad \text { if } \quad y(u)<(r+s) \lambda(h)
\end{array}\right.
$$

The above result implies that $R$ is generically driven to the boundary. Thus, we will have either $R=1$ (cross-skill matching) or $R=0$, (ex post segmentation).

\subsection{Cross-skill Matching}

Let us start with the case of an efficient cross-skill matching allocation. When $y(u)>(r+s) \lambda(h)$ and $R=1$, the efficient allocation is fully characterised by the following four conditions: ${ }^{4}$

$$
\begin{gathered}
\phi=\frac{\mu \theta^{1-\alpha}(1-\eta)+(\mu-\eta) s}{\theta^{1-\alpha}(1-\mu) \eta} \\
u=\frac{s(1-\mu)}{\left(s+\theta^{1-\alpha}\right)(1-\eta)}
\end{gathered}
$$

\footnotetext{
${ }^{4}$ Arrow's generalization of Mangasarian's sufficiency theorem (Kamien and Schwartz, 1991: 222) implies that equations (33) - (36) are the necessary and sufficient conditions for an efficient steady-state solution to the dynamic optimization problem given by equation (29). Proof of uniqueness is also provided in Appendix A
} 


$$
\begin{gathered}
\left(r+s+\phi \theta^{1-\alpha}\right) \frac{\left(r+s+\alpha \theta^{1-\alpha}\right)}{\left(r+s+\theta^{1-\alpha}\right)}=\frac{\eta(r+s)(y(u)-b)}{(1-\eta)(y(s)-y(u))} \\
\gamma\left(r+s+\alpha \theta^{1-\alpha}\right)=(1-\alpha) \theta^{-\alpha}(y(u)-b)
\end{gathered}
$$

Proposition 2 An efficient steady state allocation with cross-skill matching can be summarized by a tuplet $\left\{\theta_{S P}, \phi_{S P}, \eta_{S P}, u_{S P}\right\}$ that solve conditions $(33)-(36)$.

\section{Proof: Appendix A}

Equations (35) and (36) are, respectively, the efficient equal-value condition and the optimality condition for the mass of skilled jobs, while (33) and (34) coincide with eqs. (15) and (16). To characterise the efficiency properties of a cross-skill matching equilibrium we can therefore limit attention to eqs. (21) and (22) for the equilibrium and (35) and (36) for the efficient allocation. It is easy to show that these conditions never coincide.

First of all, from the conditions for the mass of skilled jobs, eqs. (22) and (36), it follows immediately that $\theta_{E}=\theta_{S P}$ whenever $\beta$ is equal to $\alpha$. In the matching literature this condition is commonly referred to as the Hosios' condition. Thus, whenever the Hosios' condition is satisfied, the decentralised equilibrium generates the same number of jobs per unemployed worker as the optimal allocation. Nonetheless, plugging $\beta=\alpha$ and $\theta_{E}=\theta_{S P}$ into (21) and (35) shows that the equilibrium mix of jobs is not efficient. The latter would require that $\beta=\alpha=1$.

Proposition 3 A cross-skill matching equilibrium $\left\{\theta_{E}, \phi_{E}, \eta_{E}, u_{E}\right\}$ never coincides with an efficient allocation as defined in Proposition 2. 
Proof: Appendix B

Proposition 3 strengthens the well-known efficiency result of Hosios (1990). For an economy with homogenous agents, he shows that efficiency is attained if workers obtain a share $\beta=\alpha$ of the match surplus. The above result shows that this potential for efficiency is destroyed when agents are ex ante heterogenous.

\subsubsection{Over-creation of skilled jobs}

In general, it is notoriously difficult to characterise the efficiency properties with heterogenous agents. Nonetheless, for the case in which the bargaining strength of workers satisfies Hosios' condition, we are able to provide a complete characterisation. In particular,

Proposition 4 Under Hosios' condition, $\theta_{E}=\theta_{S P}$ but firms create too few unskilled jobs as $\phi_{E}<\phi_{S P}$.

Proof: Appendix B

The intuition behind Proposition 4 is that low-skill workers (high-skill workers) are over-valued (under-valued) in equilibrium, leading to an overcreation of skilled jobs and an under-creation of unskilled jobs. Thus, under Hosios' condition, we find that low-skill workers experience a suboptimally high rate of unemployment. Formally, let $\tilde{u}(l)_{E}$ and $\tilde{u}(l)_{S P}$ denote the unemployment rates of low-skill workers in the equilibrium and in the efficient allocation, then:

Corollary 5 Under the Hosios' condition, $\tilde{u}(l)_{E}>\tilde{u}(l)_{S P}$.

The distortion of the relative wages can be understood by looking at the expressions for the outside options and the shadow values of both types of 
workers. For instance, in the case of high-skill workers, we obtain

$$
\begin{gathered}
r U(h)=b+\beta \theta^{1-\alpha}\left\{\phi Y(h, u)+(1-\phi) Y(h, s)-\frac{r U(h)}{r+s}\right\} \\
(r+s) \lambda(h)=b+\theta^{1-\alpha}\{\phi Y(h, u)+(1-\phi) Y(h, s)-\lambda(h)\}- \\
(1-\alpha) \theta^{1-\alpha}\{(1-\eta)[\phi Y(h, u)+(1-\phi) Y(h, s)-\lambda(h)]+\eta \phi(Y(l, u)-\lambda(l))\},
\end{gathered}
$$

where $\lambda(l)$ denotes the shadow value of a low-skill worker. According to (37), the reservation wage of a high-skill worker is equal to the flow value of leisure, $b$, plus the expected capital gain from employment in either a skilled or an unskilled job. The main difference with (38) is the negligence of the congestion effects. In equation $(38),(r+s) \lambda(h)$ is equal to the flow value of leisure, $b$, plus the expected capital gain from employment, minus the congestion externality of an additional high-skill unemployed on workers of the same type (high-skill),

$$
(1-\alpha) \theta^{1-\alpha}(1-\eta)[\phi Y(h, u)+(1-\phi) Y(h, s)-\lambda(h)]
$$

and on workers of the other type (low-skilled),

$$
(1-\alpha) \theta^{1-\alpha} \eta \phi(Y(l, u)-\lambda(l))
$$

In the absence of heterogeneity, this congestion externality corresponds exactly to a share $1-\alpha$ of the expected capital gain from employment. Under Hosios' condition, the congestion externality is therefore perfectly internalised in the wage, leading to an efficient allocation.

In contrast, in our economy we have two types of workers and highskill workers congest the market for some low-skill workers, who are lessproductive. The congestion externality is therefore smaller than $1-\alpha$ of the expected match surplus of a high-skill worker. Conversely, low-skill job 
seekers congest the market for some more productive high-skill workers. For this category of workers, the congestion externality is therefore larger than $1-\alpha$ of their expected match surplus. In other words, under the Hosios' condition, the relative wage payments are distorted. All high-skill workers are under-valued (in the sense that their outside option is lower than their shadow value), while the opposite holds for low-skill workers.

Next, consider the effect on profits. In our setup, the under-valuation of high-skill workers increases the profits of all jobs in the same way, while the over-valuation of low-skill workers only affects unskilled jobs. As a result, in equilibrium, firms will create too few unskilled jobs, since they have to pay low-skill workers more than their shadow value. Furthermore, since $\theta_{E}=\theta_{S P}$ this implies that firms create a suboptimally large share of skilled jobs. ${ }^{5}$

Finally, although our model is admittedly simple, the intuition behind the distortion of the relative wages can easily be extended to an economy with $N>2$ types of agents. In such an economy, all workers with an aboveaverage expected productivity would be under-valued, while all agents with a below-average expected productivity would be over-valued.

\subsection{Ex post segmentation}

We now proceed with a characterization of the efficient allocations with ex post segmentation. In this case, it is not efficient for high-skill workers to match with unskilled vacancies because $y(u)-(r+s) \lambda(h)<0$.

An efficient ex post segmentation allocation is characterized by the vec-

\footnotetext{
${ }^{5}$ The result that firms create exactly $\theta_{E}=\theta_{S P}$ jobs per unemployed worker is due to the fact that the expected wage costs of an unskilled job equals the expected marginal productivity of applicants. To obtain this result we need to take the weighted average of workers' outside options. When we repeat this calculation for workers' shadow values (using identical weights $\eta$ and $1-\eta$ ) we see that the two quantities coincide. For details see Appendix B.
} 
tor of endogenous variables $\left\{\theta_{S P}, \phi_{S P}, \eta_{S P}, u_{S P}\right\}$ satisfying the following equations:

$$
\begin{gathered}
\phi \theta^{1-\alpha} \eta u=s(\mu-\eta u) \\
(1-\phi) \theta^{1-\alpha}(1-\eta) u=s[(1-\mu)-(1-\eta) u] \\
\gamma=\theta^{-\alpha} \eta(1-\alpha)\left[\frac{[y(u)-b]}{r+s+[\phi-(1-\alpha) \eta] \theta^{1-\alpha}}\right] \\
\gamma=\theta^{-\alpha}(1-\eta)(1-\alpha)\left[\frac{[y(s)-b]}{r+s+[(1-\phi)-(1-\alpha)(1-\eta)] \theta^{1-\alpha}}\right]
\end{gathered}
$$

Equations (39) and (40) represent the laws of motion for the state variables $u(l)$ and $u(h)$ when $R=0$. As in the decentralized equilibrium, the flow condition for low-skill workers is the same as under cross-skill matching. What changes is the flow equation for high-skill workers whose exit rate out of unemployment falls from $\theta^{1-\alpha}$ to $(1-\phi) \theta^{1-\alpha}$. Comparing the above conditions to the conditions for an ex-post segmentation equilibrium we immediately obtain the following result:

Proposition 6 An ex post segmentation equilibrium never coincides with the efficient allocation $\left\{\theta_{S P}, \phi_{S P}, \eta_{S P}, u_{S P}\right\}$ as defined by (39) - (42).

Proof: Appendix A.

For the ex post segmentation case it is difficult to get more analytical results for the efficiency properties of the equilibrium. What is feasible is to show that the equilibrium matching set of high-skill workers may be inefficient. More precisely, given the under-valuation of high-skill workers we may have cases in which this type of workers accept unskilled jobs in equilibrium while it would be efficient for them to refuse these jobs. This is the aim of the next section. 


\subsection{Matching decisions}

To illustrate the possibility of inefficient matching sets, we consider the effects of a gradual increase in $y(s)$. Suppose that the initial value for $y(s)$ is consistent with an efficient cross-skill matching allocation. From eq. (36) it follows that $\partial \theta_{S P} / \partial y(s)$ is zero. Hence, as in the decentralised equilibrium, changes in $y(s)$ do not affect the labour market tightness. The only change is an increase in the fraction of skilled jobs. As we continue to raise $y(s)$, the shadow value for high-skill workers, $\lambda(h)$, therefore continues to rise and beyond some threshold value, say $\bar{y}(s)_{S P}$, the planner will prefer to switch to ex post segmentation. From our previous discussion it is clear that this switch occurs when $y(u)-(r+s) \lambda(h)<0$. For the decentralised equilibrium we obtain a similar sequence of changes. However, since $(r+s) \lambda(h)>r U(h)$ it is obvious that the switch to an ex post segmentation equilibrium takes place at a higher value of $y(s)$. Formally, let $\bar{y}(s)_{E}$ denote the lowest value of $y(s)$ for which a cross-skill matching equilibrium is feasible:

Proposition 7 When $\beta=\alpha, \bar{y}(s)_{E}>\bar{y}(s)_{S P}$.

Proof: Appendix B.

Proposition 7 completes our characterization of the complete set of steady state allocations. Three cases can be distinguished: First, for $y(s)<\bar{y}(s)_{S P}$ we obtain an inefficient cross-skill matching equilibrium. Second, for $y(s) \in$ $\left[\bar{y}(s)_{S P}, \bar{y}(s)_{E}\right]$ we obtain a cross-skill matching equilibrium while the efficient allocation features ex post segmentation. Finally, for all $y(s) \geq \bar{y}(s)_{E}$ we obtain an inefficient ex post segmentation equilibrium. Hence, the steady state equilibrium with ex post bargaining is never efficient when agents are ex ante heterogeneous. 


\subsection{Example}

In this section we present a numerical example to illustrate the three cases distinguished above. The baseline parameters are close to the ones in Albrecht and Vroman $(2002)^{6}$. Figures 2 and 3 summarise the effect of a gradual increase in $y(s)$. Three features are noteworthy. First of all, in the region where $y(s)<\bar{y}(s)_{S P}$ the decentralised equilibrium is very close to the efficient allocation. As predicted by Proposition $4, \theta_{E}=\theta_{S P}$, and invariant to changes in $y(s)$. Furthermore, due to the large share of low-skill workers $(\mu=2 / 3), \phi_{E}$ is very close to $\phi_{S P}$. Second, in the region of the ex post segmentation equilibrium $\phi_{E}$ is again below $\phi_{S P}$, but this time we also find that $\theta_{E}<\theta_{S P}$. This latter feature explains the larger difference between $\widetilde{u}(l)_{E}$ and $\widetilde{u}(l)_{S P}$. In addition, it is important to notice that the unemployment rates of both types of workers are higher under ex post segmentation. For low-skill workers the higher unemployment rate is due to the discrete fall in $\phi$. The switch from a cross-skill matching to an ex post segmentation equilibrium is therefore accompanied by a an increase in $\widetilde{u}(l)$ and a fall in $U(l)$. In contrast, for high-skill workers the higher unemployment rate is the net-effect of two opposite forces. On the one hand $\widetilde{u}(h)$ tends to increase because high-skill workers no longer match with unskilled jobs, while unemployment tends to decrease as firms are willing to open more skilled jobs. As can be observed in the figure, the first effect tends to dominate. Moreover, despite the increase in the unemployment rate, high-skill workers enjoy a discrete jump in their lifetime income due to the larger fraction of skilled jobs. Finally, for the region where $y(s) \in\left[\bar{y}(s)_{S P}, \bar{y}(s)_{E}\right]$ we obtain $\theta_{E}<\theta_{S P}$ and $\phi_{E}>\phi_{S P}$. The explanation for this last result is the sharp drop in $\phi_{S P}$ due to the change in the efficient allocation from cross-skill matching to ex-post segmentation. Once $y(s)$ reaches $\bar{y}(s)_{E}$, the equilibrium allocation features a similar drop

\footnotetext{
${ }^{6}$ Parameter configuration: $r=0.02 ; s(u)=s(s)=0.20 ; b=0.10 ; \gamma=0.40 ; y(u)=1$; $\alpha=\beta=0.50 ; \mu=2 / 3 ; y(s) \in[1.5,2.5]$
} 
in the share of low-skill jobs and $\phi_{E}$ becomes again smaller than $\phi_{S P}$.
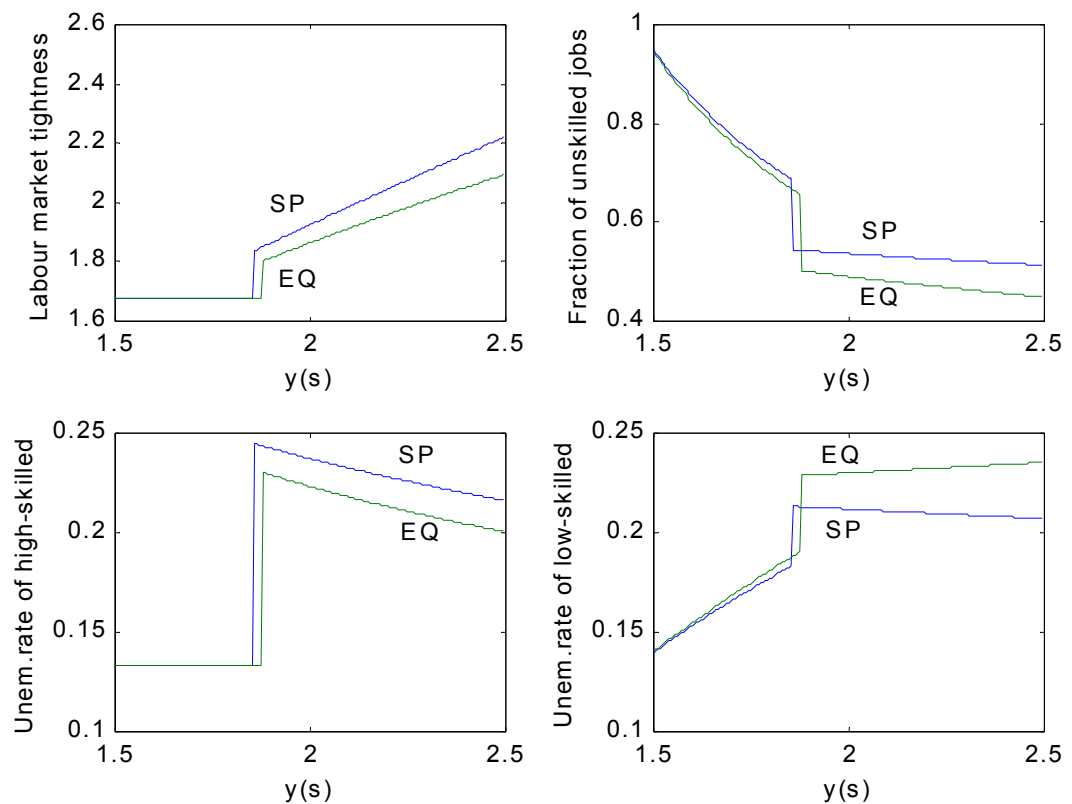

Figure 2: Efficient vs decentralised allocations
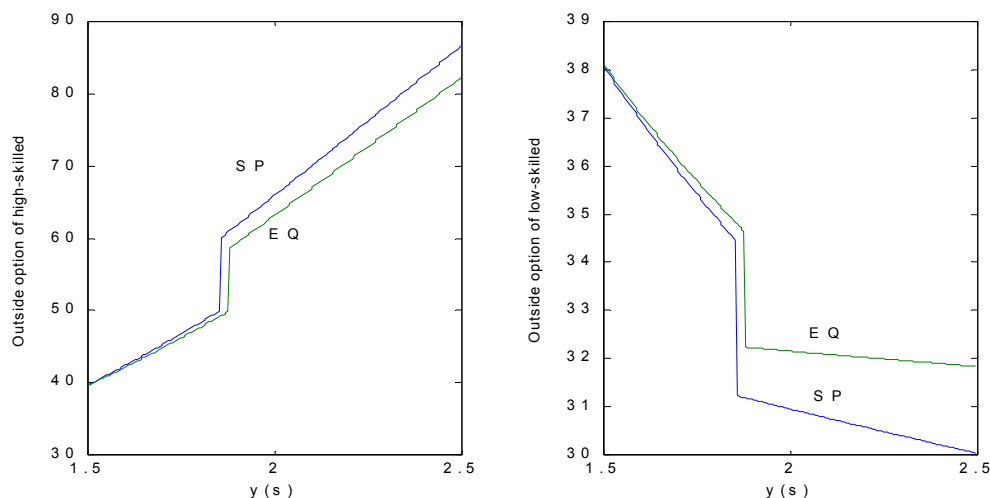

Figure 3: Outside options vs shadow values 


\section{Extensions}

In the previous section we showed that Hosios' condition leads to an excess supply of skilled jobs. This achieved our first goal. Namely to show that the competition for "over-qualified" workers may harm low-skill workers. However, the over-creation of good jobs seems to contradict the concern of policy makers that labour markets generate too many low-quality jobs.

The aim of this section is twofold. First of all we demonstrate that efficiency can be restored by taxation. And, secondly, we show that the market can generate too many unskilled jobs when workers' bargaining strength violates Hosios' condition.

\subsection{Policy implications}

In this section we return to the case of a cross-skill matching equilibrium and we show that efficiency can be restored through taxation. The instrument that we consider is a lump sum tax or subsidy on unemployed workers. Other more realistic tax schemes might achieve the same result. ${ }^{7}$ However, the taxation scheme that we illustrate below reinforces the intuition on the undervaluation (overvaluation) of high-skill (low-skill) workers that we provided in Section 4.

Formally, let $\tau(h)$ and $\tau(l)$ denote, respectively, the taxes on unemployed high-skill and low-skill workers, which in principle may be positive (taxes) or negative (subsidies). To simplify the analysis we ignore the use of the net tax proceeds. ${ }^{8}$ The new expressions of the expected lifetime income of

\footnotetext{
${ }^{7}$ One example is a hiring tax for skilled jobs. This tax can restore efficiency as it reduces the expected profits from skilled jobs. Furthermore, the hiring tax would have different distributional implications than the search taxes and subsidies considered here.

${ }^{8}$ It is straightforward to introduce a balanced government budget. To avoid distortions the government should redistribute the tax proceeds through a lump sum payment of size $\bar{\tau}=u(l) \cdot \tau(l)+u(h) \cdot \tau(h) \lessgtr 0$ to all workers.
} 
unemployed workers then become as follows:

$$
\begin{gathered}
r U(h)=b-\tau(h)+\theta^{1-\alpha}\{\phi[W(h, u)-U(h)]+(1-\phi)[W(h, s)-U(h)]\} \\
r U(l)=b-\tau(l)+\theta^{1-\alpha} \phi[W(l, u)-U(l)]
\end{gathered}
$$

The decentralized equilibrium can be solved in the same way as in the model without taxes. We make use of the condition, $V(s)=V(u)=0$ to derive the first two equations that characterize the solution, and we obtain the remaining two equations from the flow conditions.

Proposition 8 For $\beta=\alpha$ the cross-skill matching equilibrium becomes efficient under the tax scheme $\left\{\tau^{*}(l), \tau^{*}(h)\right\}$ that satisfies

$$
\begin{gathered}
\tau^{*}(l)=\frac{(1-\alpha)\left(1-\phi_{S P}\right)\left(\theta_{S P}\right)^{1-\alpha}\left(1-\eta_{S P}\right)(y(s)-y(u))}{\eta_{S P}\left[r+s+\left(\theta_{S P}\right)^{1-\alpha}\right]} \\
\tau^{*}(h)=-\frac{(1-\alpha)\left(1-\phi_{S P}\right)\left(\theta_{S P}\right)^{1-\alpha}(y(s)-y(u))}{\left[r+s+\left(\theta_{S P}\right)^{1-\alpha}\right]}
\end{gathered}
$$

Proof: Appendix B.

In line with our conclusions in Section 4.1, Proposition 8 implies that governments should impose a tax on low-skill job seekers. The size of this tax is proportional to the reduction in the mass of skilled jobs due to an additional low-skill job seeker times the productivity differential $y(s)-y(u)$. Conversely, high-skill job seekers should receive a subsidy proportional to the reduction in the matching rate of workers due to an additional low-skill job seeker times the share of skilled jobs $\left(1-\phi_{S P}\right)$ times the productivity differential $y(s)-y(u)$. When the tax scheme satisfies (43) and (44), the congestion externalities are therefore perfectly internalised. 


\subsection{Changes in the bargaining power}

To conclude our analysis we now consider the effect of different bargaining strengths. In our model, changes in the bargaining power of workers have two effects. First, as in a standard matching model the profits of jobs are a decreasing function of $\beta$. The equilibrium labour market tightness is therefore negatively correlated with $\beta$. Second, changes in workers' bargaining strength affect the relative profits of both types of jobs and this results in changes in $\phi_{E}$. We can prove that this relation between $\phi_{E}$ and $\beta$ is non-linear:

Proposition 9 The share of skilled jobs $1-\phi_{E}$ follows an inverted $U$ pattern in $\beta$. It achieves a maximum value $1-\phi_{E}>1-\phi_{S P}$ when workers' bargaining strength satisfies Hosios' condition.

Proof: Appendix B.

Since $1-\phi_{E}$ is increasing for $\beta<\alpha$ and decreasing for $\beta>\alpha$, the following corollary is immediate:

Corollary 10 Over-creation of unskilled jobs $\left(\phi_{E}>\phi_{S P}\right)$ may occur for sufficiently low or high values of $\beta$.

The intuition behind Corollary 10 is easy to explain for the case $\beta>\alpha$. In this case workers appropriate a suboptimally large share of the match surplus. Given that the skilled jobs are more valuable, this reduces the relative profits of skilled jobs leading to a reduction in $1-\phi_{E}$. Other things equal, an increase in $\beta$ therefore improves the matching rate of low-skill workers.

To obtain the overall effect on the unemployment rates of both types of workers we need to combine the changes in $\theta_{E}$ and $\phi_{E}$. For high-skill workers this results in a positive relationship between $\widetilde{u}(h)$ and $\beta$ as $\partial \theta_{E} / \partial \beta<0$. Instead, for low-skill workers the effect is ambiguous. Nonetheless, our numerical results seem to indicate that $\partial \widetilde{u}(l) / \partial \beta>0$. 
Table 1.Comparative statics of changes in $\beta$

\begin{tabular}{ccccccc}
\hline \hline & $\theta$ & $\phi$ & $\eta$ & $\widetilde{u}$ & $\widetilde{u}(h)$ & $\widetilde{u}(l)$ \\
\hline & 1.68 & 0.948 & 0.676 & 0.138 & 0.133 & 0.140 \\
\hline$\beta=0.30$ & 3.82 & 0.989 & 0.669 & 0.093 & 0.092 & 0.093 \\
$\beta=0.40$ & 2.50 & 0.955 & 0.675 & 0.115 & 0.112 & 0.116 \\
$\beta=0.50$ & 1.68 & 0.944 & 0.677 & 0.138 & 0.133 & 0.140 \\
$\beta=0.60$ & 1.11 & 0.953 & 0.675 & 0.163 & 0.159 & 0.165 \\
$\beta=0.70$ & 0.70 & 0.988 & 0.668 & 0.194 & 0.193 & 0.195 \\
\hline
\end{tabular}

Parameter configuration: $r=0.02 ; s(u)=s(s)=0.20 ; b=0.10 ; \gamma=$ $0.40 ; y(u)=1 ; y(s)=1.5 ; \alpha=0.50 ; \mu=2 / 3 .^{9}$

Table 1 reports the comparative static effects of changes in $\beta$. The first row represents the efficient allocation. Inspection of the table shows that this allocation is almost identical to the decentralised equilibrium for $\beta=0.5$. The only difference is the small over-creation of skilled jobs $\left(\phi_{E}=0.944\right.$ versus $\left.\phi_{S P}=0.948\right)$. When Hosios' condition is satisfied, the decentralised equilibrium is therefore nearly efficient. In contrast, for values of $\beta$ above (below) $\alpha$, we obtain an over-creation of unskilled jobs combined with a suboptimally low (high) value for $\theta$. Furthermore, for low-skill workers the fall in $\theta$ dominates the increase in $\phi_{E}$. At a higher value of $\beta$ low-skill workers, therefore, experience a higher unemployment rate $\widetilde{u}(l)$. Our numerical results yield a clear testable prediction about the relationship between the unemployment rate, the labour share and the degree of skill-mismatch. That is, in economies with a high labour share we should observe a high unemployment rate, a low share of skilled jobs and a large amount of high-skill workers employed on unskilled jobs. ${ }^{10}$ Over-education is documented to be a prominent

\footnotetext{
${ }^{9}$ The benchmark values are very close to the values in Albrecht and Vroman (2002).

${ }^{10}$ According to our results overeducation should also be prominent in economies with a very low labour share. In this case the explanation is less straightforward. Apparently, in
} 
feature in European labour markets (e.g. Eurostat (2003)). However, so far this phenomenon has not been related to the size of the labour share.

\section{Concluding Remarks}

In this paper we characterised the efficiency of the resource allocation in a matching model with heterogeneous agents. The conceptual framework is taken from Albrecht and Vroman (2002) and we show that the equilibrium allocation with ex post bargaining is never efficient. In particular, when workers' bargaining strength satisfies Hosios' condition, low-skill workers are overvalued in equilibrium and as a result firms create too few unskilled jobs.

This comparison between workers' shadow value and their expected lifetime income is the main innovation of this paper. It provides an intuitive explanation for the inefficiency with ex post bargaining. Moreover, although the model is admittedly simple, the results can be generalised to more complex environments with more than two types of workers and firms and/or different production technologies.

economies with an over-creation of jobs, $\theta_{E}>\theta_{S P}$, firms prefer to create unskilled jobs to limit the risk of skill-mismatch. 


\section{Appendix}

\subsection{Appendix A (Efficient Allocation)}

\section{Proof Proposition 2: Social Planner Problem}

Let $H_{c} \equiv H e^{r t}$ be the "Current-value Hamiltonian" associated with maximization problem (29).

$$
\begin{gathered}
H_{c}=\theta_{t}^{1-\alpha}\left[u(h)_{t}\left[R_{t} \phi_{t} Y(h, u)+\left(1-\phi_{t}\right) Y(h, s)\right]+u(l)_{t} \phi_{t} Y(l, u)\right]+ \\
+b\left(u(h)_{t}+u(l)_{t}\right)-\gamma \theta_{t}\left(u(h)_{t}+u(l)_{t}\right)+\lambda(l)_{t}\left[s\left(1-\mu-u(l)_{t}\right)-\phi_{t} \theta_{t}^{1-\alpha} u(l)_{t}\right]+ \\
+\lambda(h)_{t}\left[s\left(\mu-u(h)_{t}\right)-\theta_{t}^{1-\alpha} u(h)_{t}\left(1-\phi_{t}+R_{t} \phi_{t}\right)\right]
\end{gathered}
$$

Henceforth, we concentrate on the steady state, suppressing the time dependence of variables. There are three types of necessary first order conditions. The first one is the optimality condition for matching between high-skill workers and unskilled vacancies, given by $\frac{\partial H_{c}}{\partial R}$. Taking derivatives of $H_{c}$ with respect to $R$, we have:

$$
\frac{\partial H_{c}}{\partial R}=\phi \theta^{1-\alpha} u(h)[Y(h, u)-\lambda(h)]
$$

The optimal matching rule, therefore, depends on the sign of $[y(u)-(r+s) \lambda(h)]$, as specified in condition (32) in the main text.

The remaining conditions that characterize the steady state solution of this problem are:

$$
\begin{gathered}
\frac{\partial H_{c}}{\partial u(l)}=r \lambda(l) ; \frac{\partial H_{c}}{\partial u(h)}=r \lambda(h) ; \frac{\partial H_{c}}{\partial v(u)}=0 ; \frac{\partial H_{c}}{\partial v(s)}=0 \\
\frac{\partial H_{c}}{\partial \lambda(l)}=\dot{u}(l)=0 ; \quad \frac{\partial H_{c}}{\partial \lambda(h)}=\dot{u}(h)=0
\end{gathered}
$$




\section{Cross-skill matching $(R=1)$}

Under cross-skill matching, the conditions in (45) become:

$$
\begin{gathered}
(r+s) \lambda(l)=b+\phi \theta^{1-\alpha}[Y(l, u)-\lambda(l)]- \\
-(1-\alpha) \theta^{1-\alpha}\{(1-\eta)[\phi[Y(h, u)-\lambda(h)]+(1-\phi)[Y(h, s)-\lambda(h)]+ \\
+\eta \phi[Y(l, u)-\lambda(l)]\} \\
(r+s) \lambda(h)=b+\theta^{1-\alpha}[\phi[Y(h, u)-\lambda(h)]+(1-\phi)[Y(h, s)-\lambda(h)]]- \\
-(1-\alpha) \theta^{1-\alpha}\{(1-\eta)[\phi[Y(h, u)-\lambda(h)]+(1-\phi)[Y(h, s)-\lambda(h)]]+ \\
+\eta \phi[Y(l, u)-\lambda(l)]\} \\
\gamma=\theta^{-\alpha}(1-\eta)[Y(h, s)-\lambda(h)]- \\
-\alpha \theta^{-\alpha}\{(1-\eta) \phi[Y(h, u)-\lambda(h)]+(1-\phi)[Y(h, s)-\lambda(h)]+ \\
+\eta \phi[Y(l, u)-\lambda(l)]\} \\
\gamma=\theta^{-\alpha}[(1-\eta)[Y(h, u)-\lambda(h)]+\eta[Y(l, u)-\lambda(l)]]- \\
-\alpha \theta^{-\alpha}\{(1-\eta) \phi[Y(h, u)-\lambda(h)]+(1-\phi)[Y(h, s)-\lambda(h)]+ \\
+\eta \phi[Y(l, u)-\lambda(l)]\}
\end{gathered}
$$

Combining eqs. (47) - (50) we obtain:

$$
\begin{gathered}
\left(r+s+\phi \theta^{1-\alpha}\right) \frac{\left(r+s+\alpha \theta^{1-\alpha}\right)}{\left(r+s+\theta^{1-\alpha}\right)}=\frac{\eta(r+s)(y(u)-b)}{(1-\eta)(y(s)-y(u))} \\
\gamma\left(r+s+\alpha \theta^{1-\alpha}\right)=(1-\alpha) \theta^{-\alpha}(y(u)-b)
\end{gathered}
$$

From the conditions in (46) we obtain

$$
\phi=\frac{\mu \theta^{1-\alpha}(1-\eta)+(\mu-\eta) s}{\theta^{1-\alpha}(1-\mu) \eta}
$$




$$
u=\frac{s(1-\mu)}{\left(s+\theta^{1-\alpha}\right)(1-\eta)}
$$

Ex post segmentation $(R=0)$

Similarly, under ex post segmentation, the conditions in (45) become:

$$
\begin{gathered}
(r+s) \lambda(l)=b+\phi \theta^{1-\alpha}[Y(l, u)-\lambda(l)]- \\
-(1-\alpha) \theta^{1-\alpha}\{(1-\eta)(1-\phi)[Y(h, s)-\lambda(h)]+\eta \phi[Y(l, u)-\lambda(l)]\} \\
(r+s) \lambda(h)=b+\theta^{1-\alpha}(1-\phi)[Y(h, s)-\lambda(h)]- \\
-(1-\alpha) \theta^{1-\alpha}\{(1-\eta)(1-\phi)[Y(h, s)-\lambda(h)]+\eta \phi[Y(l, u)-\lambda(l)]\} \\
\gamma=\theta^{-\alpha}(1-\eta)[Y(h, s)-\lambda(h)]- \\
-\alpha \theta^{-\alpha}\{(1-\eta)(1-\phi)[Y(h, s)-\lambda(h)]+\eta \phi[Y(l, u)-\lambda(l)]\} \\
\gamma=\theta^{-\alpha} \eta[Y(l, u)-\lambda(l)]-\alpha \theta^{-\alpha}\{(1-\eta)(1-\phi)[Y(h, s)-\lambda(h)]+ \\
+\eta \phi[Y(l, u)-\lambda(l)]\}
\end{gathered}
$$

Combining eqs. (55) - (58) we get:

$$
\begin{gathered}
\frac{\gamma}{\theta^{-\alpha}}=\eta\left[\frac{(1-\alpha)[y(u)-b]}{r+s+\phi \theta^{1-\alpha}-(1-\alpha) \eta \theta^{1-\alpha}}\right] \\
\frac{\gamma}{\theta^{-\alpha}}=(1-\eta)\left[\frac{(1-\alpha)[y(s)-b]}{r+s+(1-\phi) \theta^{1-\alpha}-(1-\alpha)(1-\eta) \theta^{1-\alpha}}\right]
\end{gathered}
$$

And, from the conditions in (46) we have:

$$
\begin{aligned}
& \phi=\frac{\mu(1-\eta) \theta^{1-\alpha}+(\mu-\eta) s}{\theta^{1-\alpha}(\eta+\mu-2 \eta \mu)} \\
& u=\frac{s(1-\mu)}{\left(s+(1-\phi) \theta^{1-\alpha}\right)(1-\eta)}
\end{aligned}
$$




\section{Changes in $R$}

Next, we show that the transition from cross-skill matching to ex post segmentation occurs as we increase the productivity in skilled jobs. We start by showing that $(r+s) \lambda(h)$ is an increasing function of $y(s)$.

The shadow value of high-skill workers, $\lambda(h)$, can be written as follows:

$$
(r+s) \lambda(h)=\frac{(r+s)(b+A)+\alpha \theta_{S P}^{1-\alpha}\left[\phi_{S P} y(u)+\left(1-\phi_{S P}\right) y(s)\right]}{r+s+\alpha \theta_{S P}^{1-\alpha}}
$$

where: $A=(1-\alpha) \theta_{S P}^{1-\alpha}\left(1-\phi_{S P}\right)(y(s)-y(u)) /\left(r+s+\theta_{S P}^{1-\alpha}\right)$.

Taking derivatives in $(63)$ with respect to $y(s)$ we have:

$$
\frac{\partial[(r+s) \lambda(h)]}{\partial y(s)}=\frac{\alpha \theta_{S P}^{1-\alpha}\left[1-\phi_{S P}-\frac{\partial \phi_{S P}}{\partial y(s)}(y(s)-y(u))\right]+(r+s) \frac{\partial A}{\partial y(s)}}{r+s+\alpha \theta_{S P}^{1-\alpha}}
$$

where

$$
\frac{\partial A}{\partial y(s)}=\left[\frac{1-\alpha}{r+s+\theta_{S P}^{1-\alpha}}\right]\left[1-\phi_{S P}-\frac{\partial \phi_{S P}}{\partial y(s)}(y(s)-y(u))\right]>0
$$

Since $\frac{\partial \phi_{S P}}{\partial y(s)}<0$ and $\left(\frac{\partial A}{\partial y(s)}\right)>0$, then $\frac{\partial[(r+s) \lambda(h)]}{\partial y(s)}>0$. Thus, for small values of $y(s)$, the efficient allocation is in the cross-skill matching case. Starting from this point, increases in $y(s)$ reduces $[Y(h, u)-\lambda(h)]$, and for a threshold value, $\bar{y}(s)_{S P}$, the efficient allocation may shifts to the ex post segmentation case. The same exercise shows that the efficient allocation may change from cross-skill matching to ex post segmentation for some minimum threshold value of $1-\mu$.

\section{Arrow's Sufficiency Theorem:}

Arrow's generalization of Mangasarian's sufficiency theorem (Kamien and Schwartz, 1991: 222) states that conditions (51) - (54) are necessary and sufficient for $\{\theta, \phi, \eta, u\}$ to be the steady-state solution to the dynamic optimization problem (29), when $R=1$, if the maximized Hamiltonian function $H^{0}$ (the Hamiltonian evaluated along $\phi^{*}$ and $\theta^{*}$, where $\phi^{*}$ and $\theta^{*}$ are given 
by eqs. (33) and (36) respectively) is concave in the variables $u(h)$ and $u(l)$ for given $\lambda(h)$ and $\lambda(l)$. This implies that the Hessian matrix specified below must be negative semidefinite:

$$
H E S=\left(\begin{array}{cc}
\frac{\partial^{2} H^{0}}{\partial^{2} u(l)} & \frac{\partial^{2} H^{0}}{\partial u(l) \partial u(h)} \\
\frac{\partial^{2} H^{0}}{\partial u(h) \partial u(l)} & \frac{\partial^{2} H^{0}}{\partial^{2} u(h)}
\end{array}\right)
$$

Taking derivatives with respect to $u(h)$ and $u(l)$ in $H^{0}$ we obtain:

$$
\begin{gathered}
\frac{\partial^{2} H^{0}}{\partial^{2} u(l)}=\frac{2 \mu(u(h))^{2}\left(s+\left(\theta^{*}\right)^{1-\alpha}\right)(y(u)-y(s))}{(1-\mu)(u(l))^{3}(r+s)}<0 \\
\frac{\partial^{2} H^{0}}{\partial^{2} u(h)}=\frac{2 \mu\left(s+\left(\theta^{*}\right)^{1-\alpha}\right)(y(u)-y(s))}{(1-\mu) u(l)(r+s)}<0 \\
\frac{\partial^{2} H^{0}}{\partial u(l) \partial u(h)}=\frac{\partial^{2} H^{0}}{\partial u(h) \partial u(l)}=\frac{-2 \mu u(h)\left(s+\left(\theta^{*}\right)^{1-\alpha}\right)(y(u)-y(s))}{(1-\mu)(u(l))^{2}(r+s)}>0
\end{gathered}
$$

Operating with the previous expressions we obtain that $|H E S|=0$. Thus, since $\frac{\partial^{2} H^{0}}{\partial^{2} u(l)}<0, \frac{\partial^{2} H^{0}}{\partial^{2} u(h)}<0$ and $|H E S|=0$, the Hessian matrix is negative semidifinite which gives us the necessary and sufficient conditions for $\{\theta, \phi, \eta, u\}$ to be a steady-state solution for the dynamic optimization problem (29) when high-skill workers accept unskilled job offers ${ }^{11}$. Nonetheless, since the Hessian matrix is negative semidifinite, this does not guarantee uniqueness.

\section{Uniqueness of the cross-skill matching efficient allocation}

From equation (52), we know that $\partial \theta_{S P} / \partial \mu=0$. Using this result, we establish uniqueness by showing that $d(1-\phi) / d(1-\mu)<0$.

\footnotetext{
${ }^{11}$ The Hessian matrix is not very difficult to be computed for the cross-skill matching case. However, applying Arrow's generalization of Mangasarian's sufficiency theorem to the dynamic optimization problem (29) when $R=0$ (ex post segmentation case), would require computing the Hessian matrix numerically.
} 
From equation (53) we obtain:

$$
\frac{\eta}{1-\eta}=\frac{\mu\left(s+\theta^{1-\alpha}\right)}{(1-\mu)\left(s+\phi \theta^{1-\alpha}\right)}
$$

Substituting into condition (51) we have:

$$
1-\mu=\frac{\left(s+\theta^{1-\alpha}\right)(r+s)\left(r+s+\theta^{1-\alpha}\right)(y(u)-b)}{\Delta}
$$

where:

$$
\begin{gathered}
\Delta=\left(r+s+\phi \theta^{1-\alpha}\right)(y(s)-y(u))\left(r+s+\alpha \theta^{1-\alpha}\right)\left(s+\phi \theta^{1-\alpha}\right)+ \\
\left(s+\theta^{1-\alpha}\right)(r+s)\left(r+s+\theta^{1-\alpha}\right)(y(u)-b)
\end{gathered}
$$

The existence of the cross-skill matching efficient allocation implies that there exist a solution for (65). Furthermore equation (65) is continuously differentiable, allowing the use of the Implicit Function Theorem. Thus, taking the total derivative in $(65)$ :

$\frac{d(1-\phi)}{d(1-\mu)}=\left\{-\left[\frac{\left(s+\theta^{1-\alpha}\right)(r+s)\left(r+s+\theta^{1-\alpha}\right)(y(u)-b)}{\Delta^{2}}\right] \frac{\partial \Delta}{\partial(1-\phi)}\right\}^{-1}$

where:

$$
\frac{\partial \Delta}{\partial(1-\phi)}=-(y(s)-y(u))\left(r+s+\alpha \theta^{1-\alpha}\right) \theta^{1-\alpha}\left[r+2\left(s+\phi \theta^{1-\alpha}\right)\right]<0
$$

Uniqueness of the cross-skill matching efficient allocation is proved recursively. First, from equation (52) we derive a unique value of $\theta$. Second, from (66) and (67), $(1-\phi)$ defines a strictly increasing function of $(1-\mu)$. Thus, for any value of $(1-\mu)$ we get a unique value of $(1-\phi)$. Given unique values of $\theta$ and $(1-\phi)$, from equation (51) we obtain a unique value of $\eta$. Finally, given unique values of $\theta$ and $\eta$, equation (54) defines a unique value for $u$. 


\subsection{Appendix B (Proofs of main results)}

\section{Proof Proposition 3: Inefficient cross-skill matching equilibrium}

When $\beta=\alpha$, from eqs. (22) and (36) we have $\theta_{E}=\theta_{S P}$. Plugging this result into eqs. (21) and (35) we obtain:

$$
\begin{gathered}
\left(r+s+\beta \phi_{E} \theta_{S P}^{1-\alpha}\right)=\frac{\eta_{E}(r+s)(y(u)-b)}{\left(1-\eta_{E}\right)(y(s)-y(u))} \\
\left(r+s+\phi_{S P} \theta_{S P}^{1-\alpha}\right) \frac{\left(r+s+\alpha \theta_{S P}^{1-\alpha}\right)}{\left(r+s+\theta_{S P}^{1-\alpha}\right)}=\frac{\eta_{S P}(r+s)(y(u)-b)}{\left(1-\eta_{S P}\right)(y(s)-y(u))}
\end{gathered}
$$

From (68) and (69) it follows that $\beta=\alpha=1$ is needed for a cross-skill matching equilibrium to be efficient.

\section{Proof Proposition 4}

1. Overcreation of skilled jobs:

Plugging $\beta=\alpha$, and $\theta_{E}=\theta_{S P}$ into (15), (16) and (21) we can derive the following expression:

$$
\frac{\mu(r+s)(y(u)-b)}{(1-\mu)(y(s)-y(u))}=\frac{\left(s+\phi_{E} \theta_{S P}^{1-\alpha}\right)}{\left(s+\theta_{S P}^{1-\alpha}\right)}\left(r+s+\alpha \phi_{E} \theta_{S P}^{1-\alpha}\right)
$$

Next, proceeding in the same way for the efficient allocation, we combine eqs (33), (34) and (35) which yields:

$$
\begin{gathered}
\frac{\mu(r+s)(y(u)-b)}{(1-\mu)(y(s)-y(u))}=\frac{\left(s+\phi_{S P} \theta_{S P}^{1-\alpha}\right)\left(r+s+\phi_{S P} \theta_{S P}^{1-\alpha}\right)\left(r+s+\alpha \theta_{S P}^{1-\alpha}\right)}{\left(s+\theta_{S P}^{1-\alpha}\right)\left(r+s+\theta_{S P}^{1-\alpha}\right)} \\
<\frac{\left(s+\phi_{S P} \theta_{S P}^{1-\alpha}\right)\left(r+s+\alpha \phi_{S P} \theta_{S P}^{1-\alpha}\right)}{\left(s+\theta_{S P}^{1-\alpha}\right)}
\end{gathered}
$$

The term on the left-hand side of eqs. (70) and (71) is the same, therefore, we can derive the following inequality:

$$
\left(s+\phi_{E} \theta_{S P}^{1-\alpha}\right)\left(r+s+\alpha \phi_{E} \theta_{S P}^{1-\alpha}\right)<\left(s+\phi_{S P} \theta_{S P}^{1-\alpha}\right)\left(r+s+\alpha \phi_{S P} \theta_{S P}^{1-\alpha}\right)
$$


In order for (72) to hold, it must be the case that $\phi_{E}<\phi_{S P}$. As $\partial \phi / \partial \eta<0$, then we have $\eta_{E}>\eta_{S P}$.

2. Undervaluation (overvaluation) of high-skill (low-skill) workers:

Substituting $\beta=\alpha\left(\theta_{E}=\theta_{S P}\right)$ and the values of $J(h, s)$ and $w(h, s)$ into the free entry condition for skilled vacancies we obtain:

$$
\gamma=(1-\alpha) \theta_{S P}^{-\alpha}\left(1-\eta_{E}\right)\left[\frac{y(s)-r U(h)}{r+s}\right]
$$

On the other hand, the optimality condition for $v(s)$ can be written as follows:

$$
\gamma=(1-\alpha) \theta_{S P}^{-\alpha}\left(1-\eta_{S P}\right)\left[\frac{y(s)}{r+s}-\lambda(h)\right]
$$

Combining eqs. (73) and (74) we obtain:

$$
\left(1-\eta_{E}\right)\left[\frac{y(s)-r U(h)}{r+s}\right]=\left(1-\eta_{S P}\right)\left[\frac{y(s)}{r+s}-\lambda(h)\right]
$$

When $\beta=\alpha,\left(1-\eta_{E}\right)<\left(1-\eta_{S P}\right)$, then, in order for equation (75) to hold it must be the case that $r U(h)<(r+s) \lambda(h)$ (high-skill workers are undervalued).

Similarly, substituting $\beta=\alpha\left(\theta_{E}=\theta_{S P}\right)$ and the values of $J(h, u), J(l, u)$, $w(h, u)$ and $w(l, u)$ into the free entry condition for unskilled vacancies, we have:

$$
\begin{gathered}
\gamma=(1-\alpha) \theta_{S P}^{-\alpha}\left(1-\eta_{E}\right)\left[\frac{y(s)-r U(h)}{r+s}\right]+ \\
+(1-\alpha) \theta_{S P}^{-\alpha}\left\{\eta_{E}\left[\frac{y(u)-r U(l)}{r+s}\right]-\left(1-\eta_{E}\right)\left[\frac{y(s)-y(u)}{r+s}\right]\right\}
\end{gathered}
$$

while the optimality condition for $v(u)$ can be written as follows:

$$
\begin{gathered}
\gamma=(1-\alpha) \theta_{S P}^{-\alpha}\left(1-\eta_{S P}\right)\left[\frac{y(s)}{r+s}-\lambda(h)\right]+ \\
+(1-\alpha) \theta_{S P}^{-\alpha}\left\{\eta_{S P}\left[\frac{y(u)}{r+s}-\lambda(l)\right]-\left(1-\eta_{S P}\right)\left[\frac{y(s)-y(u)}{r+s}\right]\right\}
\end{gathered}
$$


Combining eqs. (76) and (77), and plugging equation (75) into them, we have:

$$
\frac{\eta_{E}}{1-\eta_{E}}\left[\frac{y(u)-r U(l)}{r+s}\right]=\frac{\eta_{S P}}{1-\eta_{S P}}\left[\frac{y(u)}{r+s}-\lambda(l)\right]
$$

When $\beta=\alpha, \eta_{E}>\eta_{S P}$ then, in order for equation (78) to hold, it must be true that: $r U(l)>(r+s) \lambda(l)$ (low-skill workers are overvalued).

3. Identical labour market tightnesses $\left(\theta_{E}=\theta_{S P}\right)$

Making use of the expression of $r U(h)$, given by equation (37), and the analogous expression for $r U(l)$ given by:

$$
r U(l)=b+\beta \phi \theta^{1-\alpha}\left[Y(l, u)-\frac{r U(l)}{r+s}\right],
$$

the weighted average of workers' outside options can be written as:

$$
\begin{aligned}
& \eta r U(l)+(1-\eta) r U(h)=b+\beta \theta^{1-\alpha}\left\{\eta \phi\left[Y(l, u)-\frac{r U(l)}{r+s}\right]+\right. \\
& \left.\quad(1-\eta)\left[\phi Y(h, u)+(1-\phi) Y(h, s)-\frac{r U(h)}{r+s}\right]\right\}
\end{aligned}
$$

Following the same reasoning, we use the expression of $\lambda(h)$, given by equation (38), and the expression of $\lambda(l)$ given by:

$$
\begin{aligned}
& \lambda(l)=b+\phi \theta^{1-\alpha}[Y(l, u)-\lambda(l)]- \\
& (1-\alpha) \theta^{1-\alpha}\{(1-\eta)[\phi Y(h, u)+(1-\phi) Y(h, s)-\lambda(h)]+\eta \phi(Y(l, u)-\lambda(l))\},
\end{aligned}
$$

to derive the following expression for the weighted average of workers' shadow values:

$$
\begin{aligned}
& \eta(r+s) \lambda(l)+(1-\eta)(r+s) \lambda(h)=b+\alpha \theta^{1-\alpha}\{\eta \phi[Y(l, u)-\lambda(l)]+ \\
& (1-\eta)[\phi Y(h, u)+(1-\phi) Y(h, s)-\lambda(h)]\}
\end{aligned}
$$

Thus, for given $\eta(\phi)$, the two quantities, (80) and (82) coincide when the Hosios' condition is satisfied. 
4. Corollary 5: $\left(\beta=\alpha \Longrightarrow \widetilde{u}(l)_{E}>\widetilde{u}(l)_{S P}\right)$

From (36) we know that changes in $\mu$ do not affect $\theta_{S P}$, so an increase in $\mu$ implies that, for a given $\phi_{S P}, u(l)$ will increase. All else equal, this increases $\eta_{S P}$, so the right-hand side of equation (35) will increase, while the left-hand side will decrease. Thus, for equation (35) to hold, $\phi_{S P}$ has to increase. Summarizing, increasing $\mu$ makes the creation of low-skill vacancies more attractive, resulting in an increase of $\phi_{S P}$. From this, it is easy to show that $\widetilde{u}(l)_{S P}=s /\left[s+\phi_{S P} \theta_{S P}^{1-\alpha}\right]$ is a decreasing function of $\mu$.

Proof Proposition 6: Inefficient ex post segmentation equilibrium

Comparing eqs. (27) and (28) with eqs. (41) and (42), it can be derived that we need $\beta=\alpha=1$ to get efficiency.

\section{Proof Proposition 7: Matching decisions}

From Appendix A we know that $\partial[(r+s) \lambda(h)] / \partial y(s)>0$. Thus, to demonstrate that $\bar{y}(s)_{E}>\bar{y}(s)_{S P}$, we first need to show that $r U(h)$ is an increasing functions of $y(s)$. Taking derivatives of $r U(h)$ with respect to $y(s)$ we obtain:

$$
\frac{\partial[r U(h)]}{\partial y(s)}=\frac{\beta \theta_{E}^{1-\alpha}\left[1-\phi_{E}-\frac{\partial \phi_{E}}{\partial y(s)}(y(s)-y(u))\right]}{r+s+\beta \theta_{E}^{1-\alpha}}>0
$$

since $\partial \phi_{E} / \partial y(s)=\left(\partial \phi_{E} / \partial \eta_{E}\right)\left(\partial \eta_{E} / \partial y(s)\right)<0$.

Thus, $r U(h)$ and $(r+s) \lambda(h)$ are monotonically increasing functions of $y(s)$. Moreover, by Proposition 4, we know that $(r+s) \lambda(h)>r U(h) \forall y(s)$. Thus, it follows that the switch to an ex post segmentation equilibrium takes place at a higher value of $y(s)$, that is, $\bar{y}(s)_{E}>\bar{y}(s)_{S P}$.

\section{Proof Proposition 8: Optimal tax scheme}

Under the tax scheme $\{\tau(l), \tau(h)\} \in \Re^{2}$, the expressions for the outside options of both types of workers are the following: 


$$
\begin{gathered}
r U(h)=b-\tau(h)+\theta^{1-\alpha}\{\phi[W(h, u)-U(h)]+(1-\phi)[W(h, s)-U(h)]\} \\
r U(l)=b-\tau(l)+\theta^{1-\alpha} \phi[W(l, u)-U(l)]
\end{gathered}
$$

Substituting $r U(h)$ and $r U(l)$ into the free entry conditions for skilled and unskilled vacancies, and making use of the equal value condition $V(s)=V(u)$ we obtain the following expression:

$$
\left(1-\eta_{E}\right)\left[r+s+\phi_{E} \theta_{E}^{1-\alpha}\right](y(s)-y(u))=\eta_{E}(r+s)(y(u)-b+\tau(l))
$$

Plugging equation (84) into the free entry condition of skilled jobs we obtain:

$$
\gamma\left[r+s+\beta \theta_{E}^{1-\alpha}\right]=(1-\beta) \theta_{E}^{-\alpha}\left[y(u)-b+\eta_{E} \tau(l)+\left(1-\eta_{E}\right) \tau(h)\right]
$$

First, comparing eqs. (36) and (85), when $\beta=\alpha$, we need

$$
\tau(l)=-\left(1-\eta_{E}\right) \tau(h) / \eta_{E}
$$

so that $\theta_{E}=\theta_{S P}=\theta$. And second, by comparing eqs. (35) and (84) the optimal value, $\tau^{*}(l)$, that makes the cross-skill matching equilibrium efficient is given by:

$$
\tau^{*}(l)=\frac{(1-\alpha)\left(1-\phi_{S P}\right) \theta_{S P}^{1-\alpha}\left(1-\eta_{S P}\right)(y(s)-y(u))}{\eta_{S P}\left[r+s+\theta_{S P}^{1-\alpha}\right]}
$$

Substituting (87) into (86), we derive the following expression for $\tau^{*}(h)$ :

$$
\tau^{*}(h)=-\frac{(1-\alpha)\left(1-\phi_{S P}\right) \theta_{S P}^{1-\alpha}(y(s)-y(u))}{\left[r+s+\theta_{S P}^{1-\alpha}\right]}
$$




\section{Proof Proposition 9: Overcreation of unskilled jobs}

Taking derivatives with respect to $\beta$ in both sides of equation (22) we obtain:

$$
\frac{\partial \theta_{E}}{\partial \beta}=-\frac{\theta_{E}\left[r+s+\theta_{E}^{1-\alpha}\right]}{(1-\beta)\left[(r+s) \alpha+\beta \theta_{E}^{1-\alpha}\right]}<0
$$

which shows that $\theta_{E}$ is monotonically decreasing in $\beta$.

Similarly, taking derivatives with respect to $\beta$ in equation (21) we have:

$$
\frac{\partial \eta_{E}}{\partial \beta}=\frac{\eta_{E}\left(1-\eta_{E}\right)\left[\phi_{E} \theta_{E}^{1-\alpha}+\beta \phi_{E}(1-\alpha) \theta_{E}^{-\alpha}\left(\frac{\partial \theta_{E}}{\partial \beta}\right)\right]}{r+s+\beta \phi_{E} \theta_{E}^{1-\alpha}}
$$

Substituting equation (89), into the previous expression, we obtain:

$$
\frac{\partial \eta_{E}}{\partial \beta}=\left[\frac{\eta_{E}\left(1-\eta_{E}\right) \phi_{E} \theta_{E}^{1-\alpha}}{r+s+\beta \phi_{E} \theta_{E}^{1-\alpha}}\right]\left[\frac{(\alpha-\beta)\left(r+s+\beta \theta_{E}^{1-\alpha}\right)}{(1-\beta)\left((r+s) \alpha+\beta \theta_{E}^{1-\alpha}\right)}\right]
$$

Finally, for a given $\theta_{E}$, taking derivatives with respect to $\beta$ in equation (15) yields:

$$
\frac{\partial \phi_{E}}{\partial \beta}=-\frac{\partial \eta_{E}}{\partial \beta}\left[\frac{\left(s+\theta_{E}^{1-\alpha}\right) \mu}{(1-\mu) \theta_{E}^{1-\alpha} \eta_{E}^{2}}\right]
$$

Therefore, from eqs. (90) and (91) we can derive the following result:

$$
\begin{aligned}
& \alpha=\beta \Rightarrow \frac{\partial \eta_{E}}{\partial \beta}=0 ; \quad \frac{\partial \phi_{E}}{\partial \beta}=0 \\
& \alpha>\beta \Rightarrow \frac{\partial \eta_{E}}{\partial \beta}>0 ; \quad \frac{\partial \phi_{E}}{\partial \beta}<0 \\
& \alpha<\beta \Rightarrow \frac{\partial \eta_{E}}{\partial \beta}<0 ; \quad \frac{\partial \phi_{E}}{\partial \beta}>0
\end{aligned}
$$

From this, it follows that $\phi_{E}>\phi_{S P}$ may occur for sufficiently low or high values of $\beta$ (Corollary 10). 


\subsection{Appendix C}
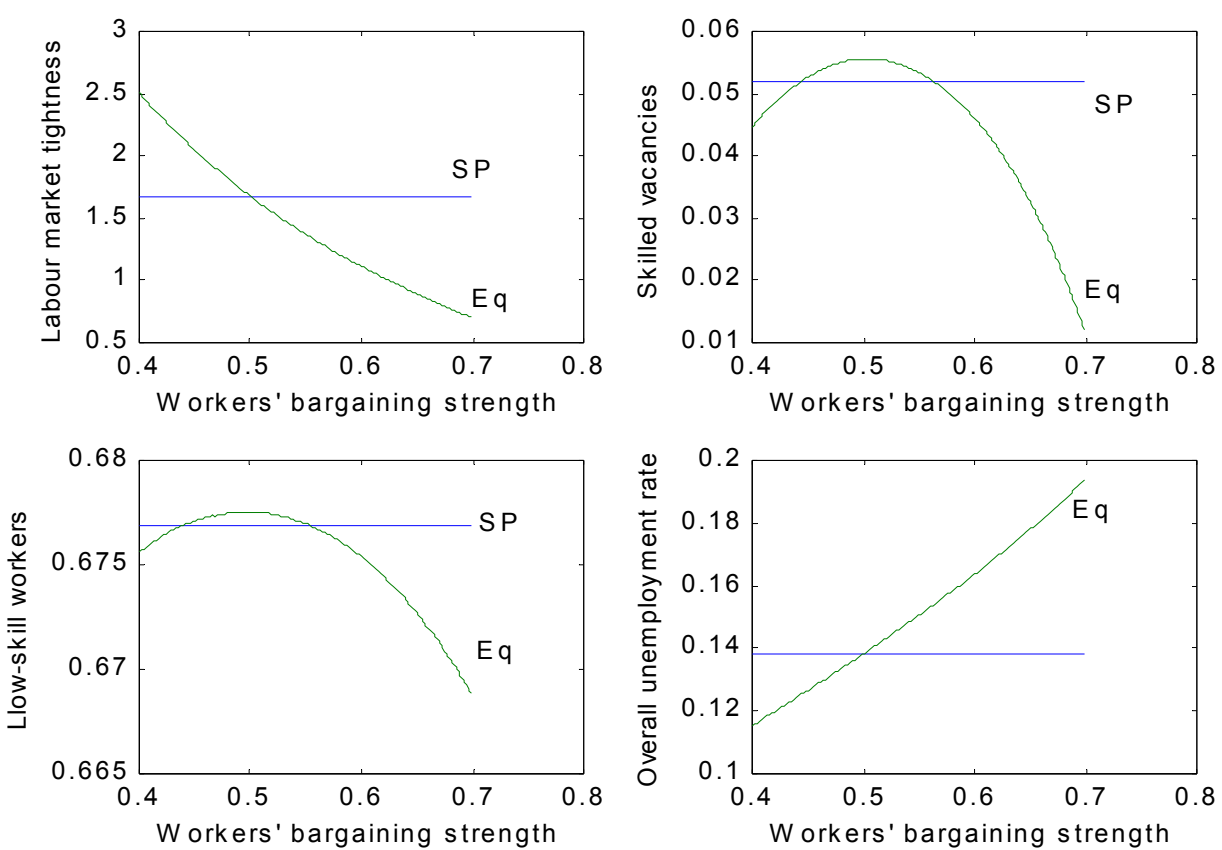

Figure 4: Comparative statics of changes in $\beta$ 


\section{References}

[1] ACEMOGLU, D., AND R. SHIMER, "Holdups and Efficiency with Search Frictions", International Economic Review, 1999, 40(4), 827-849.

[2] ALBRECHT, J., AND S.VROMAN, "A Matching Model with Endogenous Skill Requirements", International Economic Review 43 (2002), 283-305.

[3] BURDETT, K., AND M. COLES, "Long-Term Partnership Formation: Marriage and Employment", The Economic Journal, 109 (1999), F307F334.

[4] DIAMOND, P., "Aggregate Demand Management in Search Equilibrium", Journal of Political Economy, 1982, 90(5), 881-894.

[5] DOLADO, J.J., JANSEN, M., AND J.F. JIMENO, “A Matching Model of Crowding-Out and On-the-Job Search (With and Application to Spain)", CEPR Discussion Paper No. 3466.

[6] HOSIOS, ARTHUR, "On the Efficiency of Matching and Related Models of Search and Unemployment", Review of Economic Studies, 1990, 57(2), 279-298.

[7] KAMIEN, M., AND N. SCHWARTZ, Dynamic Optimization, 2nd ed. (New York: Elsevier Science Publishing, 1991).

[8] MOEN, ESPEN R., "Competitive Search Equilibrium", Journal of Political Economy, 1997, vol 105, no. 2. 
[9] MORTEnSEN, D.T., "Property Rights and Efficiency in Mating, Racing, and Related Games", American Economic Review, 1982, 72(5), 968-979.

[10] MORTEnSEN, D.T., AND C. PISSARIDES, "Job Creation and Job Destruction in the Theory of Unemployment", Review of Economic Studies, 61 (1994), 397-415.

[11] PISSARIDES, C., Equilibrium Unemployment Theory, 2nd edition (Cambridge, MA: MIT Press, 2000).

[12] SATTINGER., M., "Search and the Efficient Assignment of Workers to Jobs". International Economic Review, vol 36 (2), (May 1995), 283-302.

[13] SHIMER., R., AND L. SMITH, "Matching, Search and Heterogeneity", Advances-in-Macroeconomics, 1(1), 2001 a.

[14] SHIMER., R., AND L. SMITH, "Nonstationary Search" (2001)

[15] SHIMER., R., AND L. SMITH, "Assortative Matching and Search", Econometrica, 2000, 68(2), 343-370. 